\title{
Development of kfps Bright Flash Neutron Imaging for Rapid, Transient Processes
}

\author{
R. Zboray ${ }^{1, a^{\star}}$ Ch. Lani ${ }^{1, b}$, A. Portanova ${ }^{2, c}$ \\ ${ }^{1}$ Department of Mechanical and Nuclear Engineering, The Pennsylvania State University, 233 \\ Reber Building, University Park, PA 16802, USA \\ ${ }^{2}$ Radiation Science and Engineering Center, College of Engineering, 101 Breazeale Nuclear \\ Reactor, The Pennsylvania State University, University Park, PA 16802, USA
}

arzz65@psu.edu, bchadlani17@gmail.com, carh6@psu.edu

\begin{abstract}
Keywords: Bright Flash Neutron Radiography, Time-Resolved Neutron Imaging, kfps, TRIGA Pulse
\end{abstract}

\begin{abstract}
High-speed neutron radiography is limited by the available flux even on the strongest spallation sources. Therefore, capturing rapid, transient processes by neutron imaging remains difficult. TRIGA reactors have the capability due to their special fuel composition to produce extremely bright neutron pulses for a short duration. This opens the possibility to image short, very rapid transient processes at very high rates. We have developed bright flash thermal neutron radiography at the beam line of the 1 MW Penn State Breazeale research reactor and demonstrated imaging rates up to $4 \mathrm{kfps}$. Here we discuss and analyze some aspects of the technique.
\end{abstract}

\section{Introduction}

High temporal resolution neutron radiography is limited by the available flux at beam lines even on the strongest neutron sources like ILL, HFIR, J-PARC (typically up to $1 \times 10^{8} \mathrm{n} / \mathrm{cm}^{2} / \mathrm{s}$ ). At several hundred frames per second (fps) or even kfps rates and the corresponding exposure times high neutron flux is needed to obtain images with acceptable signal-to-noise ratio and statistics. The flux limitation can be alleviated using phase-lock, ensemble averaging techniques for periodic, repeating processes as it has been demonstrated on several samples like engines, pumps etc. However, capturing rapid, transient processes by neutron imaging remains difficult as truly high frame rates are required to avoid significant motion blur. At high enough frame rate, the motion artifacts decrease, and the corresponding image blurring can be minimized.

High frame rate imaging has been proven feasible by Zboray and Trtik up to $800 \mathrm{fps}$ [1] on the ICON beam line of the SINQ spallation source. Such frame rate was achieved at the cost of reducing the field of view (FOV) due to partial chip read out. Nakamura et al. [2] imaged at 125 fps and 500 fps the process of molten-fuel behavior by injecting liquid metal into water relevant for severe accident scenarios in nuclear reactors. Sibamoto et al. [3] were looking into water injected into molten metal as potential heat exchange and removal modality for next generation molten metal cooled reactors at 1125 fps. Pulses created in TRIGA nuclear reactors might overcome the flux limitations and offer the possibility of high-speed radiography. Such pulses can produce huge power and neutron flux surges that exceed the levels available at the strongest available spallation sources or steady reactor-based beam lines. This flux can potentially enable imaging rates at a few kilo frames per second (kfps). As the pulse duration is typically around 20-40 milliseconds, the technique is suitable to applications for examining very rapid, transient processes at such times scales. Pulsed imaging has been looked at before back in the 1980's by Bossi et al. utilizing an analog film-based system at Oregon State University [4]. They claim that it was possible to take images with frame rates up to $10 \mathrm{kfps}$, although the paper contains only 
images taken at 1 and 2 kfps. Related to Bossi's work, Wang used the same TRIGA reactor to obtain the necessary flux and used analog film to capture the images at $1000 \mathrm{fps}$ [5]. More recent work with bright flash imaging was performed by Tremsin et al. [6] and Lerche et al. [7]. However, they only imaged static objects using the high neutron flux of the pulsed McClellan Nuclear Research Center's TRIGA reactor instead of looking into dynamic processes.

We have recently presented bright flash neutron radiography utilizing pulses from the Breazeale TRIGA reactor at the Pennsylvania State University with up to $4 \mathrm{kfps}$ imaging rate [8]. First test results on a simple adiabatic, air-water two-phase flow in a bubbler demonstrated the capabilities of the technique. It can be potentially useful for any fast-transient process where one can leverage the penetrating power of neutrons through high-Z materials and their sensitivity for low-Z materials. Note that imaging somewhat slower transient processes needing only a few hundred fps can also benefit from the high flux in the pulses by having better image quality. Here we discuss and analyze further some aspects of the technique including signal-to-noise ratio, applicability of different scintillators and obtaining quantitative results for the process examined.

\section{Imaging setup, pulses}

The experimental setup is described in detail in [8], we repeat here the most important figures. The imaging beam port of the Penn State Breazeale, 1 MW TRIGA type, reactor was used. We have imaged air-water two-phase flow in an aluminum, flat bubbler with $5 \mathrm{~mm}$ internal thickness (in beam direction). The neutron imaging beam line features a tangential collimator and a steady neutron flux of $1.7 \times 10^{7} \mathrm{n} / \mathrm{cm}^{2} \mathrm{~s}$ at full power. It has an L/D collimation ratio of around 150 at the sample position. Two types of pulses, one with a 2 \$ and another with a 2.5 \$ reactivity worth, were used for the experiments (for the definition of reactivity in \$ see [9]). The former has a peak value of $284.0 \pm 6.006 \mathrm{MW}$ and the full width at half maximum (FWHM) of $24.47 \pm 2.573$ msec, while the latter has a peak value of 761.0 $\pm 12.44 \mathrm{MW}$ and a FWHM of $15.38 \pm 0.2727$ msec. The $2 \$$ pulse results in a peak neutron flux of about $4.75 \times 10^{9} \mathrm{n} / \mathrm{cm} 2$-s, whereas the peak flux from the $2.5 \$$ pulse was estimated to be about $1.3 \times 10^{10} \mathrm{n} / \mathrm{cm} 2-\mathrm{s}$. During the imaging experiments using the pulses, the neutron flux was also measured independent of the imaging system using a boron coated, electrically compensated ion chamber (CIC) detector from Westinghouse [10] placed adjacent the reactor core. The typical 2 \$ and 2.5 \$ pulses measured by the CIC and by the imaging detector are shown on the right of Fig. 1. For the imaging detector, the time evolution of the gray intensity of the image averaged over its whole FOV is shown. It illustrates that the CIC measurement can be nicely fitted with the solution of a theoretical model of Fuchs-Nordheim [9] and the normalized pulse shape from the imaging detector fits nicely to the one of CIC except at low power ( $\leq 10 \%$ pulse height). We attribute this to degraded camera performance at low signal-to-noise ratio (SNR) and high internal gain setting. The deviation from CIC decreases for $2 \mathrm{kfps}$, i.e. for higher SNR, as is shown on the same figure. We discard showing and using data from such a low SNR parts of the pulses later in the paper (see Fig. 3) the data being not trustworthy.

We have used a $400 \mu \mathrm{m}$ thick $\mathrm{LiF} / \mathrm{ZnS}$ :Cu screen from Scintacor [11] on the camera-based imaging detector setup, which featured a legacy, CMOS camera Photron FASTCAM-Ultima 512. The camera's chip has 512 x 512 pixels and can be readout up to $30 \mathrm{kfps}$, with full frame read out up to $2 \mathrm{kfps}$. The camera has a variable internal gain, which has been set to second highest level (x4) during the experiments. The camera was affixed to a Fujinon f/0 .7 lens with a focal length of $50 \mathrm{~mm}$ resulting in a field of view of ca. 70 x $70 \mathrm{~mm}$. We elaborate here on the applicability of ZnS scintillator for very high-speed imaging. Note that [11] specifies $85 \mu \mathrm{s}$ as decay time to $10 \%$ of the light output ( $\lambda=\ln 2 / 85 \mathrm{us}$ ), therefore the afterglow might have a nonnegligible effect at a few kfps imaging rate. We have setup a simple afterglow model assuming a 
single decay constant for the scintillation light, giving the afterglow fraction, AF, in the measured intensity as:

$$
A F(i)=\frac{\frac{[I(i-1)+A F(i-1) I(i-1)](1-\exp (-\lambda d t))}{\lambda d t}}{I(i)},
$$
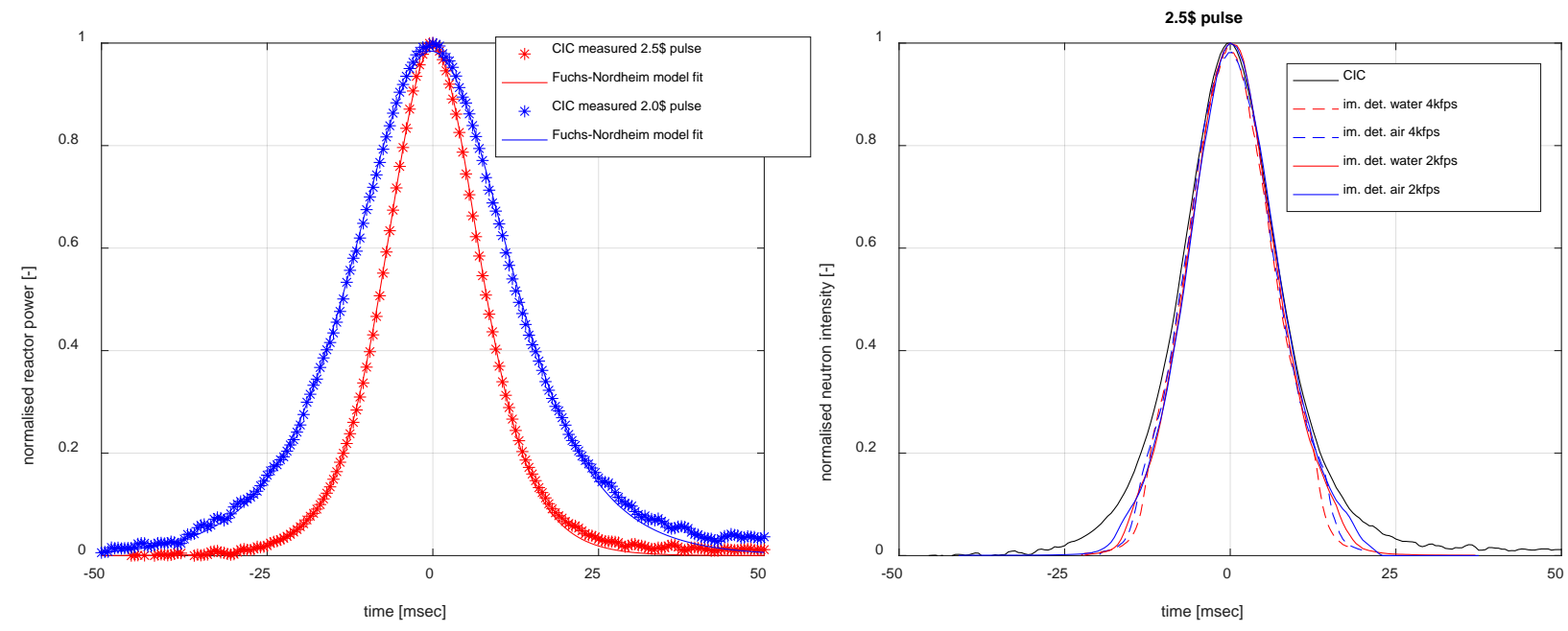

Fig. 1: (a) Fitting the pulse shape measured by the CIC detector for $2 \$$ and $2.5 \$$ pulses using the theoretical model of Fuchs-Nordheim [9]. (b) The normalized pulse shape measured by the imaging detector (with the bubbler filled with water or air, respectively) agrees well with the one obtained by CIC except at very low power.
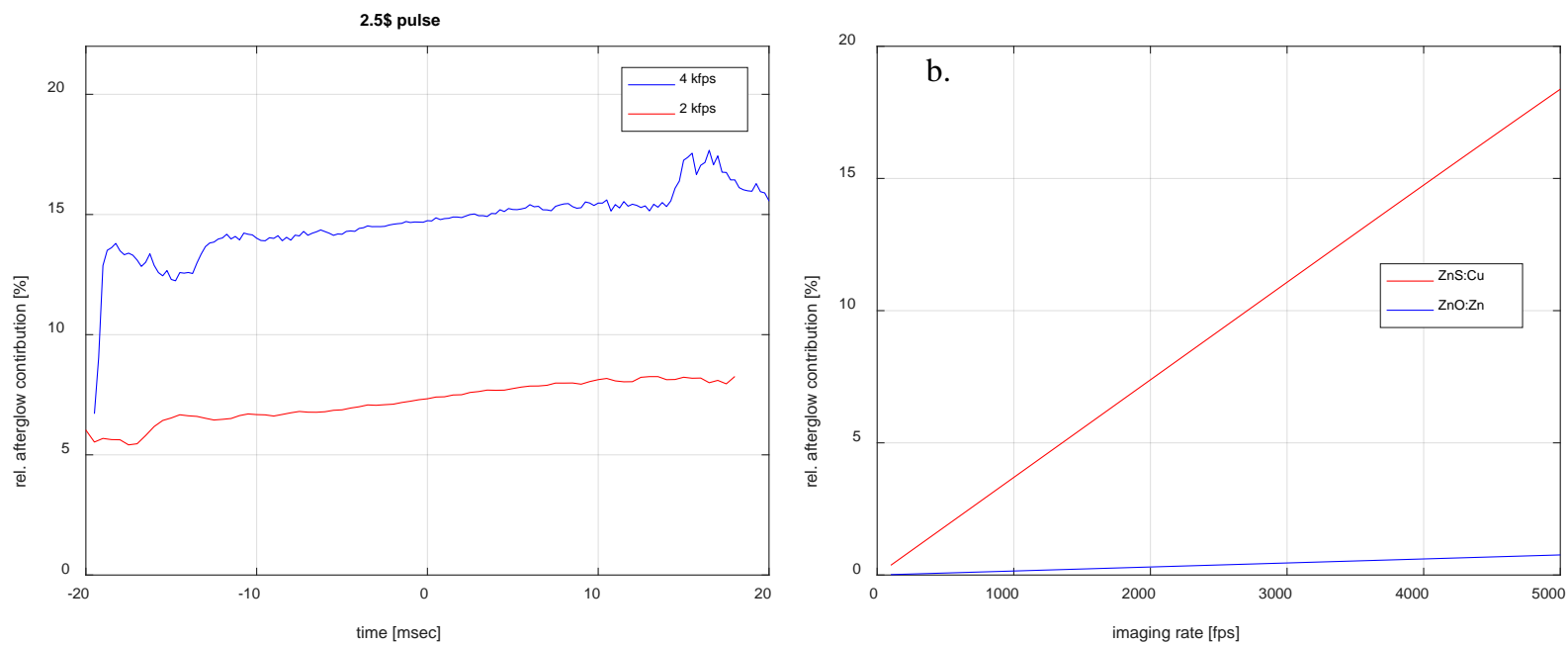

Fig. 2: (a) Fraction of the afterglow in the total measured intensity for a 2.5\$ pulse (see [8]) and two different imaging rates for our ZnS:Cu screen given by Eq. (1). (b) The equilibrium fraction of afterglow at constant imaging intensity for ZnS:Cu and ZnO:Zn scintillators as given by Eq.

(3).

where $d t$ is the exposure time and $I(i)$ is the true recorded image intensity (i.e. without afterglow) at time step $i$. For bright flash imaging, the latter changes during the pulse. The afterglow contribution in a ZnS screen is indeed not negligible around 14\% on average for 4kfps and decreases to around 6-7\% for $2 \mathrm{kfps}$ as is shown in Fig. 2a. Note that the leading half of the pulse has a slightly lower afterglow fraction than the trailing half as is expected. For the simpler case of a constant true image intensity, Eq. (1) simplifies to 


$$
A F(i)=\frac{(1+A F(i-1))(1-\exp (-\lambda d t))}{\lambda d t}
$$

and it can be easily shown that this recursive equation after a few iterations (images) settles to an equilibrium value given by

$$
A F_{e q}=\frac{1-\exp (-\lambda d t)}{\lambda d t+\exp (-\lambda d t)-1}
$$

For future work, it might be worth considering ultra-low afterglow scintillator screens like e.g. LiF/ZnO:Zn offered by Scintacor with a decay time of 3.5 us [11]. Comparison of the equilibrium afterglow fraction for $\mathrm{ZnS}: \mathrm{Cu}$ and $\mathrm{ZnO}: \mathrm{Zn}$ are shown in Fig. 2b confirming values below $1 \%$ for $\mathrm{ZnO}: \mathrm{Zn}$ even at 4-5kfps. However, as is shown in [12, 13], $\mathrm{ZnO}: \mathrm{Zn}$ has significantly lower scintillation efficiency and higher gamma sensitivity than ZnS. Therefore, the $\mathrm{LiF} / \mathrm{ZnS}$ scintillator screen still represents a reasonable compromise for a few kfps image rate.

\section{Quantitative results}

Besides the visualization of the process, one might want to extract quantitative information from the images such as the instantaneous, pixel-wise gas volume fraction distribution for the case of the two-phase flow in the bubbler. For this the images have to be normalized using corresponding flat field images taken with the bubbler filled with water and with air as:

$$
\varepsilon(x, y)=\frac{\ln \left(\frac{I_{2 p h}(x, y)}{I_{W}(x, y)}\right)}{\ln \left(\frac{I_{g}(x, y)}{I_{W}(x, y)}\right)}
$$
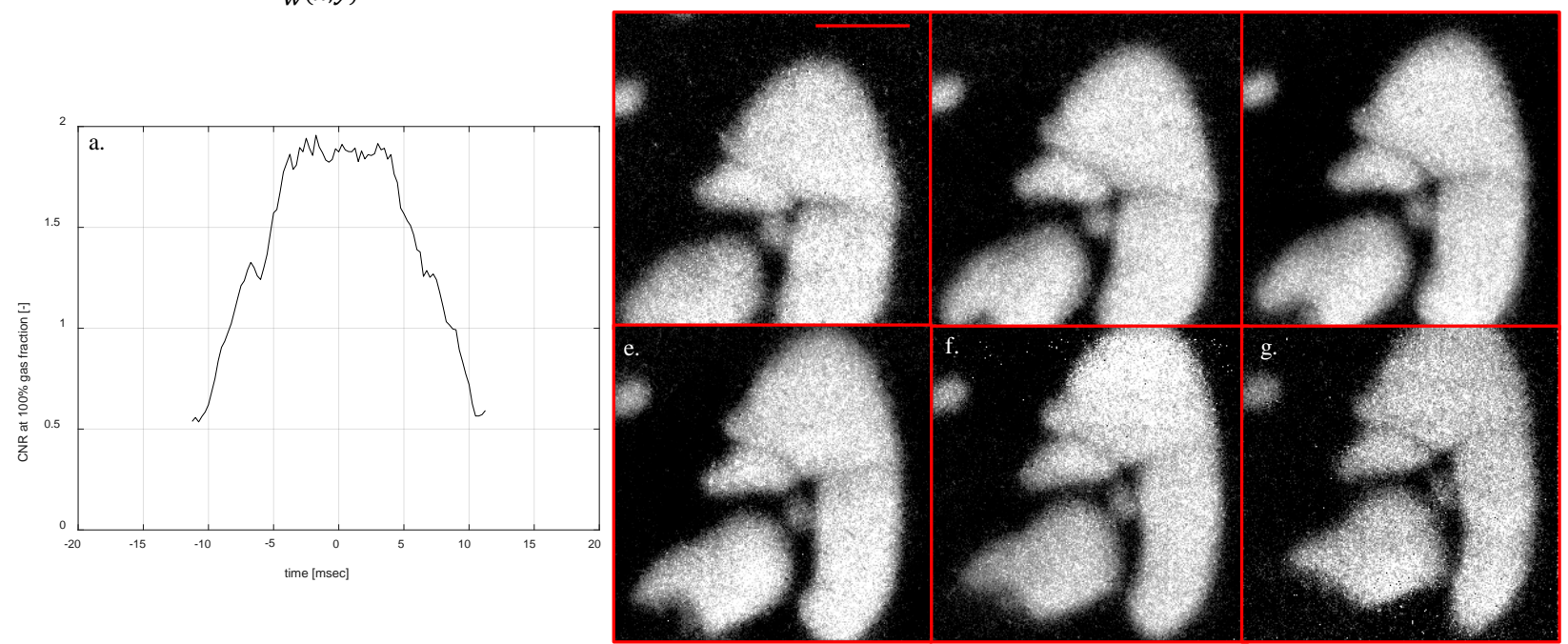

Fig. 3: (a) The variation in the CNR for $100 \%$ gas fraction for a $2.5 \$$ pulse acquired at $4 \mathrm{kfps}$. (b) to $(g)$ Corresponding image sequence of the instantaneous gas volume fraction distribution, $\varepsilon$, shown for every $10^{\text {th }}$ image taken, i.e. with a time gap of $2.5 \mathrm{~ms}$. The gray scale of the images is [-0.5,1.5] and the frame are colored in red for a better visualization.

where $I(x . y)$ is the intensity of the image at pixel $(x, y), \varepsilon$ is gas volume fraction and the subscripts 2ph stands for two-phase flow, $w$ and $g$ are for flat field images with the bubbler filled with water and with air, respectively. An image sequence of the instantaneous gas volume fraction distribution is shown in Fig $3 \mathrm{~b}$ to $3 \mathrm{~g}$ for a $2.5 \mathrm{\$}$ acquired at $4 \mathrm{kfps}$. Although it is not very visible on the sequence shown, the image quality changes significantly during the pulse with changing flux. Fig. 3a shows the contrast-to-noise ratio (CNR) between gas and liquid at 
$100 \%$ gas fraction for the same pulse and imaging rate. Obviously, the CNR increases for decreasing frame rate or for a higher pulse at the same acquisition rate.

The quantitative images of the instantaneous pixel-wise gas fraction distribution show nonnegligible bias, i.e. values above 1 and below 0 ranging from $-30 \%$ to $+180 \%$. This bias may be caused by physical processes such as beam hardening, sample and room scatter contributions, etc. Regarding scatter and beam hardening effect, it must be noted that in our earlier work using the same bubbler [1], although on a different beam line, we did not find such a strong bias in the gas fraction. That other beam line had a cold spectrum, this however is not expected to have a significant effect on beam hardening and sample scatter compared to our thermal beam. A more significant difference might stem from different levels of room scatter. Our beam line does not feature any evacuated neutron flight tubes, although our beam has about $2 \mathrm{~m}$ of free flight in air before reaching the sample and has a much compacter beam cave compared to the other beam line. Both these features can contribute to higher room scatter levels. A simple room scatter model assuming a constant room scatter contribution all over the image was used to try improve the results. According to the model the measured intensity of flat field images with the bubbler filled with air and water, respectively, can be written as:

$$
I_{g, m}=I_{g, o}+S \text {, and } I_{w, m}=I_{g, o} \exp (-\Sigma t)+S
$$

where $S$ is the constant room scatter contribution, subscript o denotes the true beam intensity without scatter and $t$ is the thickness of the water layer. $\Sigma$ is the effective neutron attenuation coefficient of the $5 \mathrm{~mm}$ thick water layer in the bubbler. Its value, including beam hardening and detector sensitivity effects, has been estimated to be around 3.5/cm based on Monte Carlo simulations in a fashion similar to [14]. Taking the ratio of the two above equations

$$
\frac{I_{w, m}}{I_{g, m}}=\frac{\exp (-\Sigma t)+S / I_{g, o}}{1+S / I_{g, o}}
$$

and using the measured ratio of flat field image intensities, the scattered fraction, $S / I_{g, o}$, can be determined. Typically, quite high values of $50-60 \%$ are found based on the data. This can be used in Eq. 4 to correct the measured intensities. This indeed improves the quantitative results quite a bit but does not fully eliminate the bias. Note that apart from the above correction method, room scatter can and will be diminished by relatively simple physical countermeasures on the beamline and on the imaging detector. Other potential bias effects like the low-count bias and the statistical uncertainty of the images have been analyzed in [8].

\section{Summary}

We have developed and tested a bright flash neutron radiography setup at the Penn State TRIGA reactor. We have proven using a simple two-phase flow device for illustration purposes that imaging with frame rates up to $4 \mathrm{kfps}$ are feasible. The imaging detector is shown to follow the intensity variation of the pulses well and agree with the predictions of the theory. Although $\mathrm{ZnS}$ based scintillator has a relatively long decay time, it is shown to be still a reasonable compromise for kfps imaging due mainly to the lack of high-light-yield, fast scintillators. The quantitative results revealed some bias effects very likely due to room scatter contribution, which can be diminished by countermeasures in future work.

The technique opens up applications for visualizing and quantifying any rapid, transient processes, where one can leverage the speed of imaging combined with the penetrating power of neutrons through high-Z material and their sensitivity to low-Z materials. Such processes will be explored in detail in the future. 


\section{Acknowledgement}

The authors are thankful to Dr. Jim Turso for providing the CIC measurement results.

\section{References}

[1] R. Zboray, P. Trtik, “800 fps neutron radiography of air-water two-phase flow”, MethodsX https://doi.org/10.1016/j.mex.2018.01.008

[2] H. Nakamura, Y. Sibamoto, Y. Anoda, Y. Kukita, K. Mishima \& T. Hibiki, "Visualization of Simulated Molten-Fuel Behavior in a Pressure Vessel Lower Head Using High-Frame-Rate Neutron Radiography”, Nuclear Technology, 125:2, (1999), 213-224. https://doi.org/10.13182/NT99-A2943

[3] Y. Sibamoto, Y. Kukita, H. Nakamura. "Visualization and Measurement of Subcooled Water Jet Injection into High-Temperature Melt by Using High-Frame-Rate Neutron Radiography”, Nuclear Technology, 139:3, (2002),205-220. https://doi.org/10.13182/NT02-A3314

[4] R.H. Bossi, A.H. Robinson, J.P. Barton, “High-Speed Motion Neutron Radiography,” Nucl. Technol., 59, 363, (1982). https://doi.org/10.13182/NT82-A33039

[5] S-H. Wang, “High Speed Motion Neutron Radiography of Two-Phase Flow”, Oregon State University (1981)

[6] A.S. Tremsin, M. Lerche, B. Schillinger, W. B. Feller, Bright flash neutron radiography capability of the research reactor at McClellan Nuclear Research Center, Nuclear Instruments and Methods in Physics Research A, 748 (2014), 46-53.

https://doi.org/10.1016/j.nima.2014.02.034

[7] M. Lerche, A.S. Tremsin, B. Schillinger, Bright Flash Neutron Radiography at the McClellan Nuclear Research Reactor, Physics Procedia, 69, (2015), 299-303. https://doi.org/10.1016/j.phpro.2015.07.042

[8] Ch. Lani \& R. Zboray, “Development of a high frame rate neutron imaging method for twophase flows”, Nucl. Inst. Meth. A. (2019), In Press, Corrected Proof. https://doi.org/10.1016/j.nima.2018.12.022

[9] D. L. Hetrick, Dynamics of Nuclear Reactors, University of Chicago Press, 1971

[10] Westinghouse, WL-8074 Boron coated, electrically compensated ion chamber.

[11] Scintacor, Neutron Screens, available at https://scintacor.com/wpcontent/uploads/2015/09/Datasheet-Neutron-Screens-High-Res.pdf, (accessed on July 20th, 2018)

[12] N. Kubota, M. Katagiri, K. Kamijo, H. Nanto, Evaluation of ZnS-family phosphors for neutron detectors using photon counting method, Nuclear Instruments and Methods in Physics Research A, 529, (2004), 321-324. https://doi.org/10.1016/j.nima.2004.05.004

[13] G. Jeff Sykora, Erik M. Schooneveld, Nigel J. Rhodes, “ZnO:Zn/6LiF scintillator - A low afterglow alternative to $\mathrm{ZnS}: \mathrm{Ag} / 6 \mathrm{LiF}$ for thermal neutron detection”, Nuclear Inst. and Methods in Physics Research A, 883, (2018), 75-82. https://doi.org/10.1016/j.nima.2017.11.052

[14] R. Zboray, H-M. Prasser, “Optimizing the performance of cold-neutron tomography for investigating annular flows and functional spacers in fuel rod bundles”, Nucl. Eng. Des., 260, (2013), 188-203. https://doi.org/10.1016/j.nucengdes.2013.03.026 